hep-lat/9805009

\title{
Testing improved actions for dynamical Kogut-Susskind quarks
}

\author{
Kostas Orginos \\ Department of Physics, University of Arizona, Tucson, AZ 85721, USA \\ Doug Toussaint \\ Department of Physics, University of Arizona, Tucson, AZ 85721, USA \\ and \\ Center for Computational Physics, University of Tsukuba, Ibaraki 305, Japan
}

The MILC Collaboration

\begin{abstract}
We extend tests of "Naik" and "fat link" improvements of the Kogut-Susskind quark action to full QCD simulations, and verify that the improvements previously demonstrated in the quenched approximation apply also to dynamical quark simulations. We extend the study of flavor symmetry improvement to the complete set of pions, and find that the nonlocal pions are significantly heavier than the local nonGoldstone pion. These results can be used to estimate the lattice spacing necessary for realistic simulations with this action.
\end{abstract}




\section{Introduction}

Because of the much larger computer time required to generate sample lattices with small lattice spacing in full QCD, improved actions are even more important to full QCD simulations than to quenched simulations. However, because of this same high cost of computing, the effects of improving the action have mostly been tested in the quenched approximation.

For Wilson quarks, a Symanzik improved gauge action with a clover quark action [1, 2] and a renormalization group motivated improved gauge action with the conventional Wilson quark action [3] have been used in studies of full QCD thermodynamics. These studies showed significant changes in the physics relative to simulations with the conventional action.

Studies of improvements with Kogut-Susskind quarks have been slower to start, possibly because it is less urgent to cure the $o\left(a^{2}\right)$ errors in this formulation than the $o(a)$ errors in the conventional Wilson quark action. Kogut-Susskind quarks are preferred for studies of light quark thermodynamics because of their remnant exact chiral symmetry, but studies of quantities such as the equation of state have been severely limited by lattice effects [1].

Two improvements to the Kogut-Susskind quark action have been investigated. Adding a third nearest neighbor term [5] improves the quark dispersion relation and "free field" quantities such as the Stefan-Boltzmann law on the lattice. The effects of this improvement on the quenched hadron spectrum have been studied by the MILC collaboration [6], where it was found that it improves rotational symmetry as measured by the dispersion relation of the pions, but has only small effects on flavor symmetry breaking or spin splittings of the hadrons. Dynamical high temperature studies using the Naik action with four flavors of quarks have been done by the Bielefeld group [7]. A second kind of improvement - averaging over paths in the parallel transport of the quark fields, or "fattening", does improve the flavor symmetry [8]. This can be understood as a suppression of the coupling to high momentum gluons which scatter quarks from one corner of the Brillouin zone to another[g], and this understanding has been used as a guide to further reducing flavor symmetry breaking by more extensive fattening [10]. Recently, in [11] it was shown that in $\mathrm{SU}(2)$ quenched spectroscopy the local

non-Goldstone pion becomes almost degenerate with the Goldstone pion after smoothing the gauge fields with RG "cycling".

This work explores the effects of these improvements of the Kogut-Susskind quark action, together with a Symanzik improved gauge action, on simulations with dynamical quarks. One motivation for this is to make sure that the promising results from the quenched approximation also apply to full QCD, and a second motivation is to begin developing a base of experience that can be used to estimate the quality of a physics simulation done with these actions at a particular lattice spacing, or alternatively to estimate the lattice spacing required for a simulation of some particular quality. We study improvement in rotational 
invariance diagnosed through the pion dispersion relation, and flavor symmetry restoration from the zero momentum masses of the pions. In addition to the local non-Goldstone pion, the " $\pi_{2}$ ", we look also at the nonlocal pions [12, 13]. No attempt is made at this stage to study whether these improved actions show better scaling of mass ratios.

\section{The simulations}

The gauge action used in most of these simulations was a Symanzik improved gauge action 14, [15, 16, [17. This is the same gauge action used in the MILC studies of improvement for the quenched spectrum[6].

$$
S_{G}=\left(\frac{\beta_{i m p}}{3}\right)\left(\sum_{x ; \mu<\nu}\left(P_{\mu \nu}\right)-\frac{1}{20 u_{0}^{2}}\left(1+0.4805 \alpha_{s}\right) \sum_{x ; \mu \neq \nu}\left(R_{\mu \nu}\right)-\frac{1}{u_{0}^{2}} 0.03325 \alpha_{s} \sum_{x ; \mu<\nu<\sigma}\left(C_{\mu \nu \sigma}\right)\right)
$$

where $P$ is the standard plaquette in the $\mu, \nu$-plane, and $R$ and $C$ denote the real part of the trace of the ordered product of $\mathrm{SU}(3)$ link matrices along $1 \times 2$ rectangles and $1 \times 1 \times 1$ paths, respectively. Here $\beta_{i m p}=10 / g^{2}$, and $\alpha_{s}=-4 \log \left(u_{0}\right) / 3.0684$. Because these are exploratory calculations, no effort was made to tune $u_{0}$. Instead, the value found in Ref. [6] at $\beta_{\text {imp }}=7.1, u_{0}=0.8441$ was used.

The conventional one plaquette action was used in some simulations for comparison.

$$
S_{G}=\left(\frac{\beta_{\text {conv }}}{3}\right)\left(\sum_{x ; \mu<\nu}\left(P_{\mu \nu}\right)\right)
$$

Here $\beta_{\text {conv }}=6 / g^{2}$.

The quark action used the hopping matrix $2 m+\not D$ with $\not D$ parameterized as

$$
\begin{aligned}
& \not D(x, y)=\sum_{\mu} \eta_{\mu}(x) \\
& \left(c_{1}\left(U_{\mu}(x) \delta_{y, x+\hat{\mu}}-U_{\mu}^{\dagger}(x-\hat{\mu}) \delta_{y, x-\hat{\mu}}\right)\right. \\
& +c_{3}\left(U_{\mu}(x) U_{\mu}(x+\hat{\mu}) U_{\mu}(x+2 \hat{\mu}) \delta_{y, x+3 \hat{\mu}}-\right.\text { "backwards Naik term") } \\
& +w_{3} \sum_{\nu \neq \mu}\left(\left\{U_{\nu}(x) U_{\mu}(x+\hat{\nu}) U_{\nu}^{\dagger}(x+\hat{\mu})+U_{\nu}^{\dagger}(x-\hat{\nu}) U_{\mu}(x-\hat{\nu}) U_{\nu}(x-\hat{\nu}+\hat{\mu})\right\} \delta_{y, x+\hat{\mu}}\right. \\
& \text { - "backwards staple term")) }
\end{aligned}
$$


Here $c_{1}$ is the coefficient of the conventional single-link term, $c_{3}$ is the coefficient of the third nearest neighbor (Naik) term, and $w_{3}$ is the coefficient of the "fat link", or staple term. Note that this parameterization of the fattening is designed to be convenient for full QCD updating, and differs from the parameterization used in Ref. [8].

Four different valence quark actions were used in computing the spectrum. The conventional "one-link" quark action has $c_{1}=1$ and $c_{3}=w_{3}=0$. The "Naik" action has $c_{1}=9 / 8$, $c_{3}=-1 / 24$ and $w_{3}=0$. For the "fat-link" action we arbitrarily weight each staple with one half the weight of the single link path, and normalize so that the total weight of the one link path is one: $c_{1}=1 / 4, c_{3}=0$, and $w_{3}=1 / 8$. In Ref. [8] it was found that the improvement in flavor symmetry is fairly insensitive to the exact weight of the staples. Finally, for the "one+fat+Naik" action, we keep the relative weight of the staple to the one link term at one half, but normalize the total of the single link plus six staples to $9 / 8: c_{1}=(9 / 8)(1 / 4)$, $c_{3}=-1 / 24$ and $w_{3}=(9 / 8)(1 / 8)$. Notice that we have not tadpole improved the quark action by including a factor of $1 / u_{0}^{2}$ in the third nearest neighbor coupling, as was done in Ref. [6]. This would have been reasonable for the Naik action, but for the one + fat + Naik it would have made much less difference, since the bulk of the coupling to the nearest neighbor sites also comes from three link paths, which presumably are boosted by the same factor. Here the main effect would have been a renormalization of the mass.

The gauge action and gauge force computations used a generalized gauge action code written by Tom DeGrand and Anna Hasenfratz[18]. For the inversions of the fermion matrix, the conjugate gradient algorithm was used. The products of links connecting to the third nearest neighbor sites and the sum of the single link plus staples were precomputed before starting the conjugate gradient, so the computer time per iteration was about twice that of the conventional one-link quark action. The fermion force can be computed by a straightforward generalization of the algorithm described in [19. The actions used here are all in the class of actions where the fermion matrix is $2 m$ times the identity matrix plus a $\not D$ operator which couples even sites to odd sites. For these actions the Hermitian positive definite operator $M^{\dagger} M$ is block diagonal, coupling even sites only to even sites. Thus, in the standard way, the pseudofermion action can be written without further doubling the number of flavors from four to eight as

$$
S_{F}=\phi_{e}^{\dagger} \frac{1}{M^{\dagger} M} \phi_{e}
$$

where $\phi_{e}$ is a pseudofermion defined on the even lattice sites $(x+y+z+t$ is even). (For simplicity, we sketch the force computation for four flavors of quarks with the hybrid Monte Carlo algorithm; adapting it to two flavors is done in the standard way 19. Roughly,

$$
\begin{aligned}
F_{f} & \approx \frac{\partial}{\partial U}\left\langle\phi_{e}\left|\frac{1}{M^{\dagger} M}\right| \phi_{e}\right\rangle \\
& =\left\langle\phi_{e}\left|\frac{1}{M^{\dagger} M} \frac{\partial}{\partial U}\left(M^{\dagger} M\right) \frac{1}{M^{\dagger} M}\right| \phi_{e}\right\rangle
\end{aligned}
$$




$$
=\left\langle X_{e}\left|\frac{\partial}{\partial U}\left(M^{\dagger} M\right)\right| X_{e}\right\rangle
$$

where $\phi_{e}$ is restricted to even lattice sites and $X_{e}$ is $\left(M^{\dagger} M\right)^{-1} \phi_{e}$, also restricted to even sites. The diagonal (mass) term in $M^{\dagger} M$ doesn't contribute to the force, so by defining $X_{o}$ on odd lattice sites to be $\not D X_{e}$, this can be written as

$$
\left\langle X_{o}\left|\frac{\partial M}{\partial U}\right| X_{e}\right\rangle+\left\langle X_{e}\left|\frac{\partial M^{\dagger}}{\partial U}\right| X_{o}\right\rangle .
$$

Except for a minus sign from the adjoint, the two terms can be treated identically. Now $M$ depends on a particular link matrix $U_{\mu}(x)$ through every path in the quark action which contains this link. After projecting out the traceless anti-Hermitian part, the terms where the link is going backwards $\left(U^{\dagger}\right.$ appears in $\left.M\right)$ just give a factor of two. So, with $\not \supset X_{e}$ stored in the odd sites of $X$, the force computation involves

- Compute $X_{e}=\left(M^{\dagger} M\right)^{-1} \phi_{e}$ on even sites.

- Compute $X_{o}=\not D X_{e}$ on odd sites

- For each path in the quark action, for each link going in direction $+\hat{\mu}$, calculate a contribution to the "force", or change in the momentum associated with the link:

- Parallel transport $X$ along the path from each end of the path to the site of the momentum being updated. This involves multiplying by $U$ or $U^{\dagger}$, and by the Kogut-Susskind phase factors $\eta_{\nu}(x)$.

- Take the traceless anti-Hermitian part of $|F\rangle\langle B|$, where $F$ and $B$ are $X$ transported from the forwards and backwards ends of the path.

- Multiply by -1 if the link is an odd numbered link in the path, and by -1 if you are updating the momentum on an odd site. This takes care of the minus signs coming from the adjoint in the last line of Eq. 0 .

- Since the $\eta_{\mu}$ contribute an unwanted minus sign for every plaquette enclosed by a loop, multiply by -1 for each plaquette enclosed by a loop consisting of the path being considered and some standard path. For example, when the KogutSusskind phases are absorbed into the link matrices, the staple contribution to the fat link gets a minus sign relative to the single link connection.

- Multiply by the coefficient of the path, which includes a minus sign for paths that are part of the "backwards" part of $\not D$.

Some optimizations are possible, since several different paths passing through a site may share the same forwards or backwards parts and vectors transported to one site and used in the force computation there can be subsequently transported to the next site along the 
path. The timings of the various parts of the computation depend on many factors such as the machine used, the lattice sizes, and how carefully each part is optimized. As an example, on an $8^{4}$ lattice on an alpha workstation with this improved action the gauge force computation requires 24.6 seconds, the fermion force computation 5.4 seconds and the conjugate gradient 0.183 seconds per iteration. About 400 conjugate gradient iterations were required at $\beta_{i m p}=7.5$ and $a m_{q}=0.015$.

Since getting good acceptance in the hybrid Monte Carlo algorithm is a good test of the program, some four flavor results on small lattices were done first. While these were intended just to be test runs, because they had large lattice spacing and larger quark mass than the two flavor runs they ended up showing the largest effects on the pion dispersion relation, so some of the four flavor results for the pion dispersion relation are reported. Two flavor runs with the improved action were done at $\beta_{i m p}=7.1,7.3$ and 7.5. Some of the conventional action results needed for comparison can be found in the literature, but some conventional action lattices were generated at $\beta_{\text {conv }}=5.5$. This was done to get a reasonable match of lattice spacing and lattice size to the $\beta_{i m p}=7.3$ improved action runs and to get the masses of all of the pions. For all of the sample sets that were generated, the meson spectrum was calculated with both the conventional and improved valence actions. In some cases valence actions where the Naik and the fattening were turned on separately were also used. Several local meson propagators were calculated at nonzero momenta, but the Goldstone pion gave by far the most accurate results, and our analysis of rotational symmetry will concentrate on these propagators.

\section{$3 \quad$ Results}

The largest data sets are two flavor runs at $\beta_{i m p}=7.1,7.3$ and 7.5, together with conventional action runs at $\beta_{c o n v}=5.5$ chosen to match the $\beta_{i m p}=7.3$ runs. At $\beta_{i m p}=7.1$ a single mass value was run, at $m_{\pi} / m_{\rho}=0.51$ for the conventional action and $m_{\pi} / m_{\rho}=0.57$ for the fat + Naik action. At the two larger $\beta_{i m p}$ values, two quark masses were used which bracket $m_{\pi} / m_{\rho}=0.55$, so it is possible to interpolate to this arbitrary value for comparing the different actions. Also, to compare the lattice spacings of the different runs we can use the $\rho_{2}$ mass interpolated to $m_{\pi} / m_{\rho}=0.55$ as an arbitrary length standard, and one which is more accessible than an extrapolation to the physical light quark mass. (The $\rho_{2}$ is used instead of the $\rho$ since at large lattice spacings it often gives a better signal.)

Table I contains the pion masses, including both local and nonlocal pions at zero momentum, and the Goldstone pion at momenta $\frac{2 \pi}{L}(0,0,0), \frac{2 \pi}{L}(0,0,1)$ and $\frac{2 \pi}{L}(0,1,1)$. The $\rho_{2}$ mass is also tabulated, as it will be used to set the scale.

The absence of rotational symmetry on the lattice leads to meson dispersion relations that 
differ from the $E(\vec{k})^{2}=m^{2}+\vec{k}^{2}$ expected in the continuum. The dimensionless parameter "speed of light squared", which should be one, is used to parameterize the deviations from the correct dispersion relation,

$$
c^{2}=\frac{E^{2}(\vec{k})-E^{2}(\overrightarrow{0})}{\vec{k}^{2}} .
$$

Table II shows $c^{2}$ for the Goldstone pion at the two smallest lattice momenta in selected runs. While the two flavor runs with smaller quark masses are relevant for estimating the lattice spacing required for physical simulations, improvement of the dispersion relation is naturally most dramatic in the runs where it is worst with the unimproved action, namely at large lattice spacing and large quark mass. Therefore Table II also contains a four flavor run at quark mass $a m_{q}=0.1$, corresponding to $m_{\pi} / m_{\rho}=0.76$ or 0.82 with the conventional and improved actions respectively. Ideally, all of these numbers would be quoted at the same physical momentum. A crude approximation to this was achieved by increasing the spatial lattice size in units of $a$ as $\beta$ was increased, hoping to keep the physical lattice size constant. The extent to which this was achieved is shown by expressing the unit of lattice momentum, $2 \pi / L$ in units of the $\rho_{2}$ mass interpolated to $m_{\pi} / m_{\rho}=0.55$, and is included in this table. Since $c^{2}$ involves the difference of the pion energy at zero and nonzero momentum, a jackknife analysis was done to estimate the statistical error on $c^{2}$ at $\beta=7.3$ and 7.5. Errors estimated from the jackknife analysis were not significantly different than errors estimated naively from the statistical error in the nonzero momentum pion energies. The improved action $10 / g^{2}=7.3$ and conventional action $6 / g^{2}=5.5$ results in Tables I and II contain the non-Goldstone pion masses and speed of light for the Goldstone pion with the fat-link and Naik improvements turned on separately. Within the fairly poor statistics, this is consistent with the quenched result of Ref. [6] that, as expected, it is the Naik term that is responsible for the improvement in the dispersion relation. 


\begin{tabular}{|c|c|c|c|c|c|c|c|c|c|}
\hline$\beta$ & $m_{q}$ & $N_{s}$ & $S_{V}$ & $\rho_{2}$ & $\begin{array}{l}\pi_{0} \\
\gamma_{5} \otimes \gamma_{5} \\
\gamma_{0} \gamma_{5} \otimes \gamma_{0} \gamma_{5}\end{array}$ & $\begin{array}{l}\pi_{1} \\
\gamma_{5} \otimes \gamma_{i} \gamma_{5} \\
\gamma_{0} \gamma_{5} \otimes \gamma_{i} \gamma_{j}\end{array}$ & $\begin{array}{l}\pi_{2} \\
\gamma_{5} \otimes \gamma_{i} \gamma_{0} \\
\gamma_{0} \gamma_{5} \otimes \gamma_{i}\end{array}$ & $\begin{array}{l}\pi_{3} \\
\gamma_{5} \otimes \gamma_{0} \\
\gamma_{0} \gamma_{5} \otimes \mathbf{1}\end{array}$ & $\begin{array}{l}\vec{p}_{\pi} \\
(0,0,1) \\
(0,1,1) \\
\end{array}$ \\
\hline \multirow[t]{4}{*}{$7.1_{I}$} & 0.05 & 10 & $\mathrm{OL}$ & $1.180(26)$ & $0.6033(4)$ & $1.035(8)$ & $1.170(12)$ & $1.16(7)$ & $0.831(3)$ \\
\hline & & & & & $1.013(10)$ & $1.127(25)$ & $1.25(2)$ & $1.21(3)$ & $0.980(4)$ \\
\hline & & & OFN & $1.080(14)$ & $0.6180(7)$ & $0.772(2)$ & $0.842(6)$ & $0.911(8)$ & $0.865(5)$ \\
\hline & & & & & $0.765(3)$ & $0.846(6)$ & $0.920(10)$ & $0.96(2)$ & $1.067(14)$ \\
\hline \multirow[t]{8}{*}{$7.3_{I}$} & 0.020 & 12 & $\mathrm{OL}$ & $0.861(15)$ & $0.3985(7)$ & $0.635(6)$ & $0.719(13)$ & $0.74(2)$ & $0.642(4)$ \\
\hline & & & & & $0.636(11)$ & $0.69(1)$ & $0.73(3)$ & $0.74(3)$ & $0.800(6)$ \\
\hline & & & ON & $0.81(2)$ & $0.383(1)$ & $0.620(4)$ & - & - & $0.643(4)$ \\
\hline & & & & & $0.60(1)$ & $0.67(1)$ & - & - & $0.81(1)$ \\
\hline & & & $\mathrm{OF}$ & $0.73(2)$ & $0.373(1)$ & $0.455(3)$ & - & - & $0.629(8)$ \\
\hline & & & & & $0.460(5)$ & $0.496(5)$ & - & - & $0.85(2)$ \\
\hline & & & OFN & $0.722(11)$ & $0.360(1)$ & $0.443(4)$ & $0.490(4)$ & $0.532(5)$ & $0.63(1)$ \\
\hline & & & & & $0.445(4)$ & $0.488(6)$ & $0.541(7)$ & $0.572(9)$ & $0.86(2)$ \\
\hline \multirow[t]{8}{*}{$5.5_{C}$} & 0.015 & 12 & $\mathrm{OL}$ & $0.71(1)$ & $0.3513(9)$ & $0.536(5)$ & $0.584(9)$ & $0.594(13)$ & $0.619(7)$ \\
\hline & & & & & $0.541(8)$ & $0.581(10)$ & $0.586(8)$ & $0.594(5)$ & $0.79(1)$ \\
\hline & & & ON & $0.71(1)$ & $0.341(1)$ & $0.517(4)$ & - & - & $0.619(9)$ \\
\hline & & & & & $0.53(1)$ & $0.570(10)$ & - & - & $0.81(1)$ \\
\hline & & & $\mathrm{OF}$ & $0.61(1)$ & $0.317(2)$ & $0.369(4)$ & - & - & $0.585(12)$ \\
\hline & & & & & $0.390(6)$ & $0.401(7)$ & - & - & $0.84(2)$ \\
\hline & & & OFN & $0.615(14)$ & $0.306(2)$ & $0.356(3)$ & $0.415(8)$ & $0.463(11)$ & $0.585(6)$ \\
\hline & & & & & $0.366(6)$ & $0.394(7)$ & $0.387(5)$ & $0.412(5)$ & $0.81(4)$ \\
\hline \multirow[t]{4}{*}{$7.3_{I}$} & 0.040 & 12 & $\mathrm{OL}$ & $0.988(14)$ & $0.5481(7)$ & $0.799(13)$ & $0.861(6)$ & $0.883(9)$ & $0.735(2)$ \\
\hline & & & & & $0.770(10)$ & $0.871(11)$ & $0.88(2)$ & $0.93(2)$ & $0.883(6)$ \\
\hline & & & OFN & $0.851(9)$ & $0.507(1)$ & $0.575(3)$ & $0.618(3)$ & $0.652(4)$ & $0.734(4)$ \\
\hline & & & & & $0.586(4)$ & $0.617(4)$ & $0.654(6)$ & $0.681(8)$ & $0.907(7)$ \\
\hline \multirow[t]{4}{*}{$5.5_{C}$} & 0.030 & 12 & $\mathrm{OL}$ & $\begin{array}{l}0.845(9) \\
\end{array}$ & $0.4846(6)$ & $0.701(7)$ & $0.762(10)$ & $0.785(12)$ & $\begin{array}{l}0.691(3) \\
\end{array}$ \\
\hline & & & & & $0.697(7)$ & $0.74(2)$ & $0.741(8)$ & $0.779(9)$ & $0.851(8)$ \\
\hline & & & OFN & $0.725(10)$ & $0.430(2)$ & $0.486(3)$ & $0.546(7)$ & $0.580(6)$ & $0.668(4)$ \\
\hline & & & & & $0.484(4)$ & $0.519(4)$ & $0.525(3)$ & $0.543(6)$ & $0.93(4)$ \\
\hline \multirow[t]{4}{*}{$7.5_{I}$} & 0.015 & 16 & OL & $0.701(9)$ & $0.3387(7)$ & $0.500(8)$ & $0.520(9)$ & $0.551(4)$ & $0.502(4)$ \\
\hline & & & & & $0.499(8)$ & $0.526(6)$ & $0.547(6)$ & $0.57(2)$ & $0.640(12)$ \\
\hline & & & OFN & $0.607(11)$ & $0.2892(12)$ & $0.337(2)$ & $0.369(4)$ & $0.389(4)$ & $0.484(3)$ \\
\hline & & & & & $0.336(3)$ & $0.371(4)$ & $0.389(5)$ & $0.408(5)$ & $0.62(2)$ \\
\hline \multirow[t]{4}{*}{$7.5_{I}$} & 0.030 & 16 & OL & $0.826(15)$ & $0.4680(9)$ & $0.634(9)$ & $0.676(4)$ & $0.697(3)$ & $0.604(2)$ \\
\hline & & & & & $0.621(4)$ & $0.672(6)$ & $0.687(7)$ & $0.703(7)$ & $0.713(4)$ \\
\hline & & & OFN & $0.702(8)$ & $0.4076(9)$ & $0.4552(14)$ & $0.4824(16)$ & $0.505(2)$ & $0.565(4)$ \\
\hline & & & & & $0.455(2)$ & $0.481(3)$ & $0.505(3)$ & $0.524(3)$ & $0.700(16)$ \\
\hline
\end{tabular}


Table 1: Summary of $\pi$ and $\rho_{2}$ masses from selected runs. The top two lines of the table show the format for tabulating the masses. The first column is the gauge coupling, where the subscript " $\mathrm{C}$ " indicates the conventional one-plaquette action and the subscript "I" indicates the Symanzik improved gauge action. The second column is the quark mass. The third column is the spatial lattice size, and the fourth column is the valence quark action. In this column "O" stands for the conventional "one link" action, "OFN" for the improved "one-link + fat + Naik" action. "OF" and "ON" stand for the "one-link + fat" and "one-link + Naik" actions respectively. The fifth column is the $\rho_{2}$ mass. The remaining columns are pion masses. The first four columns of pion masses correspond to distance 0,1 , 2 and 3 operators respectively, all at zero momentum. There are two pion operators at each distance, shown one above the other. These operators have the "spin $\otimes$ flavor" structures shown in the table header [12]. The Goldstone pion is the upper pion in the distance zero column. Goldstone pion energies at nonzero momenta are in the last column, labelled by the momentum in units of $2 \pi / N_{s}$. 


\begin{tabular}{llllll}
$\beta$ & $a m_{q}$ & $S_{V}$ & $\frac{2 \pi}{L m_{\rho}(0.55)}$ & $c^{2}(0,0,1)$ & $c^{2}(0,1,1)$ \\
\hline 7.1 & $0.1_{4}$ & OL & NA & $0.72(2)$ & $0.65(2)$ \\
7.1 & $0.1_{4}$ & OFN & NA & $0.98(4)$ & $0.99(2)$ \\
\hline 7.1 & 0.05 & OL & $0.53(2)$ & $0.82(1)$ & $0.75(1)$ \\
7.1 & 0.05 & OFN & $0.57(1)$ & $0.94(2)$ & $0.95(3)$ \\
\hline 7.3 & 0.02 & OL & $0.53(1)$ & $0.88(2)$ & $0.88(4)$ \\
7.3 & 0.02 & ON & - & $0.97(2)$ & $0.93(3)$ \\
7.3 & 0.02 & OF & - & $0.94(4)$ & $1.04(8)$ \\
7.3 & 0.02 & OFN & $0.64(1)$ & $0.96(5)$ & $1.09(10)$ \\
\hline 5.5 & 0.015 & OL & $0.66(1)$ & $0.95(5)$ & $0.91(4)$ \\
5.5 & 0.015 & ON & - & $0.98(5)$ & $0.97(4)$ \\
5.5 & 0.015 & OF & - & $0.88(6)$ & $1.10(9)$ \\
5.5 & 0.015 & OFN & $0.79(1)$ & $0.90(7)$ & $1.09(20)$ \\
\hline 7.3 & 0.04 & OL & $0.53(1)$ & $0.88(1)$ & $0.87(2)$ \\
7.3 & 0.04 & OFN & $0.64(1)$ & $1.03(3)$ & $1.03(2)$ \\
\hline 5.5 & 0.030 & OL & $0.66(1)$ & $0.88(2)$ & $0.89(3)$ \\
5.5 & 0.030 & OFN & $0.79(1)$ & $0.95(3)$ & $1.25(17)$ \\
\hline 7.5 & 0.015 & OL & $0.49(1)$ & $0.91(3)$ & $0.95(3)$ \\
7.5 & 0.015 & OFN & $0.60(1)$ & $0.93(5)$ & $0.95(3)$ \\
\hline 7.5 & 0.030 & OL & $0.49(1)$ & $0.92(1)$ & $0.94(3)$ \\
7.5 & 0.030 & OFN & $0.60(1)$ & $0.94(3)$ & $1.03(6)$ \\
\hline
\end{tabular}

Table 2: "Speed of light" for the Goldstone pion. The first three columns are the gauge coupling, quark mass and valence quark action. The fourth column is the unit of lattice momentum in units of the arbitrary mass scale $m_{\rho}$ at $m_{\pi} / m_{\rho}=0.55$. The last two columns are $c^{2}$ obtained from the lowest two nonzero lattice momenta. 
Another symmetry which is broken on the lattice is the flavor symmetry. The pion masses with $\beta_{i m p}=7.3$ or $\beta_{c o n v}=5.5$ with various valence quark actions in Table I show that the fattening of the link improves the flavor symmetry while the Naik term has little effect, in agreement with earlier studies in the quenched approximation [6, 8, 10]. Here we also see that the improvement extends to the one-link nonlocal pions. We can parameterize the splitting of the pions by the dimensionless quantity

$$
\delta_{2}=\frac{M_{\pi}^{2}-M_{G}^{2}}{M_{\rho}^{2}-M_{G}^{2}} .
$$

Here $M_{\pi}$ is one of the non-Goldstone pion masses, $M_{G}$ is the Goldstone pion mass, and $M_{\rho}$ is one of the local $\rho$ masses. Since the $\rho$ masses are nearly degenerate, it makes little difference which one we use. In our analysis we used the $\rho_{2}$ mass because it is usually estimated more accurately. This measure of flavor symmetry breaking differs from the quantity used in [8]. Here we use the squared masses since they are approximately linear in the quark mass and we will do some interpolating and extrapolating. Also, empirically this $\delta_{2}$ is fairly insensitive to the quark mass. The squared Goldstone pion mass is subtracted in the denominator to give more sensible behavior at large quark mass. At large $a m_{q}$ all of the meson masses are becoming degenerate, but it is still possible to use the difference between the Goldstone pion mass and the rho mass as a natural scale for flavor symmetry breaking. The local nonGoldstone pion is the easiest to measure, and one for which results with the conventional action are available in the literature. Figures 1 and 2 show $\delta_{2}$ for some of the pions as a function of lattice spacing, both for this improved action (both the dynamical and valence quark actions are improved), and for the conventional action. We show $\delta_{2}$ using the local non-Goldstone pion $\left(\gamma_{0} \gamma_{5} \otimes \gamma_{0} \gamma_{5}\right)$, which is consistently the lightest non-Goldstone pion, and the distance three $\gamma_{0} \gamma_{5} \otimes \mathbf{1}$ pion, which is usually the heaviest. Results for the other pion operators can be filled in using the values in Table I. Here we have plotted results for both conventional and improved dynamical actions in the same plot, with the valence actions in separate plots. Thus, to compare a completely conventional simulation (both valence and dynamical actions) with a simulation with both valence and dynamical actions improved, one should compare the burst and diamond in Fig. 1 1 with the octagons and squares in Fig. 2 . In these figures all of the data have been interpolated to $m_{\pi} / m_{\rho}=0.55$ except for the points at $\beta=7.1$.

Clearly, the fat+Naik action shows a significant improvement of the flavor symmetry breaking over the conventional action. This improvement is due to the "fattening" and not due to the Naik term which only affects the dispersion relation. The nonlocal pions have significantly larger splittings than those of the local $\pi_{2}$. Although their splittings get reduced along with the $\pi_{2}$ splitting, it is evident that the reduction is smaller. However, as expected, all the pions are approaching the same mass as the lattice spacing gets small. From our results, it is clear that one has to consider the nonlocal pions before making any judgment about the quality of the improved action. 


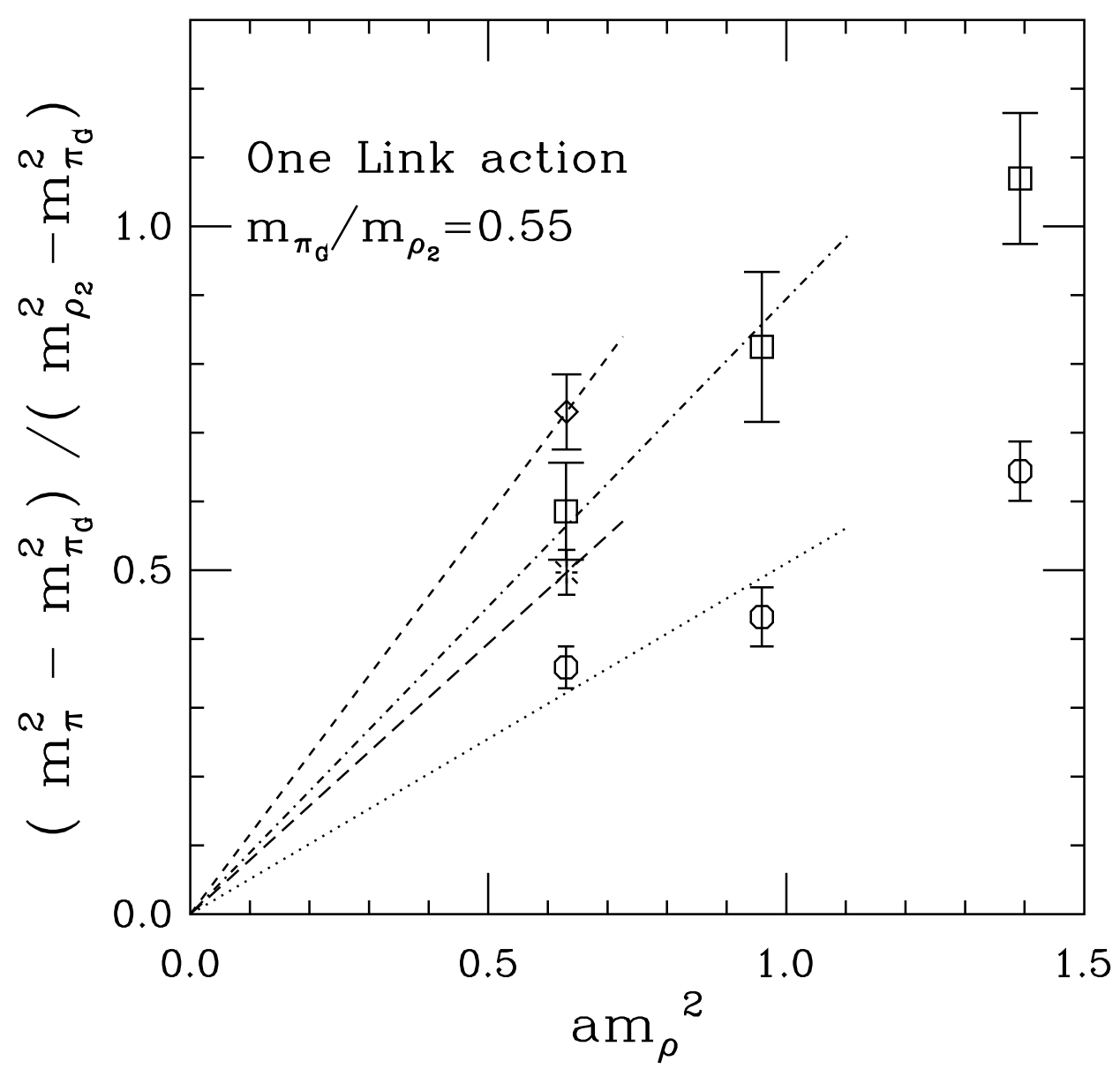

Figure 1: $\delta_{2}$ for the conventional (One Link) valence action. Points with improved dynamical action are at $\beta_{i m p}=7.1,7.3$ and 7.5. All the points are interpolated to $m_{\pi} / m_{\rho}=0.55$ except for the $\beta_{i m p}=7.1$ points for which $m_{\pi} / m_{\rho}=0.51$. The octagons correspond to the local non-Goldstone pion which has the smallest $\delta_{2}$ and the squares correspond to the three-link $\gamma_{0} \gamma_{5} \otimes 1$ pion which has the largest $\delta_{2}$. Results with the conventional dynamical action at $\beta_{\text {conv }}=5.5$ are also shown, where the burst is from the local non-Goldstone pion and the diamond from the three-link pion. The diagonal lines are crude extrapolations to smaller lattice spacing, where the dotted line is the one+fat+Naik valence action local pion, the dot-dashed line the one+fat+Naik valence action three link pion, the long dashed line the conventional valence action local pion, and the short dashed line the conventional valence action three link pion. 


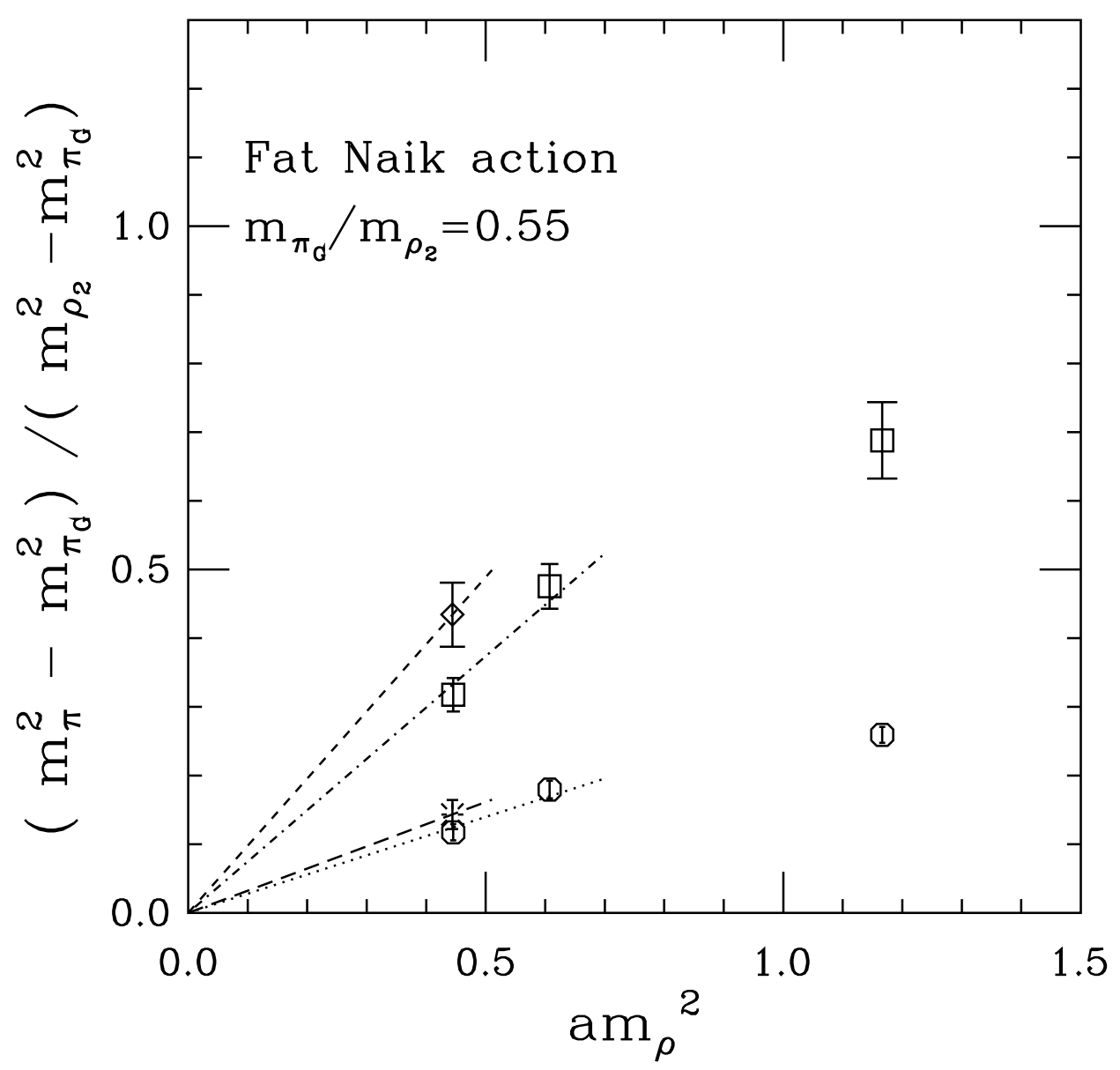

Figure 2: $\delta_{2}$ for the fat+Naik valence action. The dynamical actions and the symbols used in plotting are the same as in Fig. 1. All the points are interpolated to $m_{\pi} / m_{\rho}=0.55$ except from the the $\beta_{i m p}=7.1$ points for which $m_{\pi} / m_{\rho}=0.57$. 
Furthermore, it is evident that part of the flavor symmetry restoration is due to the improvement of the gauge action. This is also supported by the quenched spectroscopy results presented in $[\mathbb{B}]$. In the case of the one link action (Fig. 1), the improvement of the gauge action significantly reduces both the local and the nonlocal pion splittings. The local pion splittings are affected more than those of the nonlocal. In the case of the fat+Naik action (Fig. 2), we also see a reduction of all the splittings. However, the local pion splittings are improved very little while the nonlocal get a major improvement. From the above observations one may conclude that the "fattening" of the fermion action is less effective for the the nonlocal pions which they get improved mostly because of the the gauge action improvement. Further "fattening" may be needed in order to significantly improve the nonlocal pions.

\section{Conclusions}

The first conclusion is that, as expected, the improvements in rotational symmetry and flavor symmetry seen in the quenched spectrum also appear in full QCD spectrum calculations. The most serious problem for accurate simulations of full QCD with Kogut-Susskind fermions is the flavor symmetry breaking. While breaking of rotational symmetry is also serious at large lattice spacings, the light meson dispersion relations are in much better shape than the flavor symmetry. In particular, in our simulations with $\beta_{i m p}=7.5$ the speed of light for the pions is quite acceptable, while the flavor symmetry is still seriously broken.

We have extended the earlier studies of flavor symmetry breaking to include the nonlocal pions as well as the local non-Goldstone pion. The splittings between the nonlocal pions and the Goldstone pion are also reduced by improving the action, but they are significantly more massive than the local non-Goldstone pion. Worse, the amount of flavor symmetry improvement from the fat link used here is less for the nonlocal pions than the local one. This is seen in Figs. 1 and 2, where we have drawn some lines crudely indicating $\delta_{2}$ for the local pion and the 3 -link $\gamma_{0} \gamma_{5} \otimes \mathbf{1}$ pion, which is consistently the heaviest pion. Note that the slope of the line for the local pion was reduced by a larger fraction than was the slope for the 3-link pion when the valence action was improved, i.e. in going from Fig. 1 to Fig. 2 . Unfortunately, in a realistic simulation we would want all of the pions to be well behaved, so in estimating the lattice spacing required for the desired quality simulation we must consider the nonlocal pions.

An important use of action tests such as this work is to estimate the lattice parameters required to investigate real problems in QCD. An important example, and the example which partially motivated this work, is the study of the high temperature QCD phase transition. Interesting questions here include an accurate determination of the equation of state[20], and the question of whether the addition of a strange quark changes the nature of the crossover 
or phase transition 21]. To be sure that we are doing an accurate simulation in the crossover temperature range, we would like to know that our simulation is under control on both the high and low temperature sides of the crossover. The addition of the Naik term does an excellent job of improving the quark dispersion relation, and hence we expect to improve the equation of state in the high temperature phase[7]. However, the harder part of the job is to get the physics right on the low temperature side. Roughly, there the physics is a gas of thermally excited pions. To reproduce this physics, we need the pion dispersion relation to be good up to energies large compared to the temperature, and the pion masses to be nearly degenerate - that is, for flavor symmetry to be restored. For concreteness, suppose that we are interested in the effects of adding a strange quark. Viewed from the low temperature side of the transition, this adds kaons with mass $500 \mathrm{MeV}$ to the physics. To isolate this effect, we should require that all of the pions have masses small compared to this. For example, we might demand that when the Goldstone pion has its physical mass of $140 \mathrm{MeV}$, that the non-Goldstone pions have masses less than one half the kaon mass, or $250 \mathrm{MeV}$. This requires $\delta_{2} \leq 0.075$. The $\delta_{2}$ 's for the various pions are fairly insensitive to quark mass. ( In the $10 / g_{I}^{2}=7.5$ runs they are equal within errors at $a m_{q}=0.015$ and $a m_{q}=0.030$ for both the one-link and one-fat-Naik valence actions.) Neglecting dependence of $\delta_{2}$ on quark mass, it is straightforward to find the necessary value of $a m_{\rho}$ from the lines in Figs. 1 and 2, and from there to figure out the number of time slices needed to simulate a temperature of $150 \mathrm{MeV}$. Requiring that the heaviest pion mass be less than $250 \mathrm{MeV}$ when the Goldstone pion is at $140 \mathrm{MeV}$ will require about 20 time slices with the conventional action, and about 16 with the one+fat+Naik action. (Had we used the local pion in this exercise, the results would have been 16 and 10 time slices respectively.) Sixteen time slices is a disappointingly small lattice spacing, so it is important to extend these tests to actions which might further reduce flavor symmetry breaking, such as the action discussed in Ref. [10].

\section{Acknowledgements}

This work was supported by the U.S. Department of Energy under contract DE-FG03-95ER40906. Computations were done on the CPPACS pilot machine at the University of Tsukuba, the Paragon at Oak Ridge National Laboratory and the T3E at NERSC. D.T. is grateful for the hospitality of the University of Tsukuba, where this work was begun. We thank Tom DeGrand and Anna Hasenfratz for their generalized gauge force code for the molecular dynamics algorithm. We would like to thank Graham Boyd, Tom DeGrand and Urs Heller for helpful discussions.

\section{References}


[1] C.Bernard et al., Nuc. Phys. B (Proc. Suppl.) 53, 446 (1997); Phys. Rev. D 56, 5584 (1997).

[2] F.Karsch, E.Laermann, M.Oevers and P.Schmidt, Nucl. Phys. B (Proc. Suppl.) 63, 394 (1998).

[3] Y. Iwasaki, K. Kanaya, S. Sakai and T. Yoshie, Nucl. Phys. B (Proc. Suppl.) 42, 502 (1995); Y. Iwasaki, K. Kanaya, S. Kaya and T. Yoshie, Phys. Rev. Lett. 78, 179 (1997); S. Aoki et al., Nucl. Phys. B (Proc. Suppl.) 63, 397 (1998).

[4] C.Bernard et al., Phys. Rev. D 55, 6861 (1997).

[5] S. Naik, Nucl. Phys. B316 238 (1989).

[6] C. Bernard et al., hep-lat/9712010.

[7] J. Engels et al., Phys. Lett. B396, 210 (1997).

[8] T. Blum et al., Phys. Rev. D 55, 1133 (1997).

[9] P. Lepage, proceedings of "Lattice QCD on Parallel Computers" (Tsukuba, March 1997), Nucl. Phys. (Proc. Suppl.) 60A 267 (1998).

[10] J.F. Lagae and D.K. Sinclair, Nucl. Phys. (Proc. Suppl.) 63892 (1998).

[11] T. DeGrand, A. Hasenfratz and T. Kovacs, Phys. Lett. 420B, 97 (1998).

[12] M.F.L. Golterman, Nucl. Phys. B 273, 663 (1986).

[13] N. Ishizuka, M. Fukugita, H. Mino, M. Okawa and A. Ukawa, Nucl. Phys. B(Proc. Suppl.) 26, 284 (1992); E. Laermann et al., Nucl. Phys. B(Proc. Suppl.) 26, 268 (1992).

[14] K. Symanzik, in "Recent Developments in Gauge Theories", eds. G. 't Hooft et al., 313 (Plenum, New York, 1980); Nucl. Phys. B226 187 (1983).

[15] M. Lüscher and P. Weisz, Comm. Math. Phys. 9719 (1985); Phys. Lett. 158B 250 (1985).

[16] G. P. Lepage and P. B. Mackenzie, Phys. Rev. D48 2250 (1993).

[17] M. Alford, W. Dimm, G.P. Lepage, G. Hockney and P.B. Mackenzie, Phys. Lett. 361B 87 (1995).

[18] T. DeGrand, A. Hasenfratz, P. Hasenfratz and F. Niedermayer, Nucl. Phys. B454, 615 (1995).

[19] S. Gottlieb et al., Phys. Rev. D 35, 2531 (1987).

[20] C. Bernard et al., Phys. Rev. D 55, 6861 (1997). 
[21] J.B. Kogut and D.K. Sinclair, Phys. Lett. B229, 107 (1989); F.R. Brown et al., Phys. Rev. Lett. 65, 2491 (1990); Y. Iwasaki et al., Phys. Rev. D 54, 7010 (1996). 